\title{
DECAIMENTO DE TEMPERATURA EM TUBULAÇÕES PEX PARA CONDUÇÃO DE ÁGUA QUENTE
}

\author{
Temperature Decay in Pex Pipes for Hot Water Conduction \\ NASCIMENTO, César Henrique Britto ${ }^{1}$; UEHARA, Takashi ${ }^{2}$; FERREIRA, Armando Traini ${ }^{3}$ \\ Recebido em 15 de julho de 2021, aprovado em 06 de setembro de 2021, publicado em 18 de novembro de 2021
}

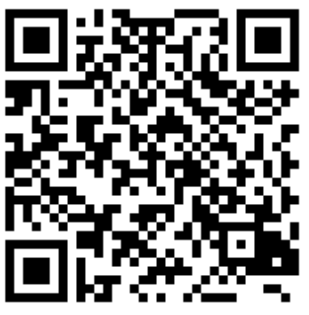

Palavras-chave:

Temperatura,

Tubulação,

PEX.

Keywords:

Temperature,

Pipe,

PEX.
RESUMO: O Polietileno Reticulado (PEX) é um material flexível utilizado no mercado da construção civil em sistemas prediais de água quente e fria, e assim como outros materiais, ao conduzir água quente, perde temperatura entre o ponto inicial e o ponto final de um trecho analisado, ocasionando perda de energia em forma de calor para o ambiente. Este artigo tem como objetivo verificar o comportamento do decaimento de temperatura em tubulações PEX de água quente de diversos diâmetros e fornecer essas informações para as fichas técnicas e os projetistas, para isto, serão adotados métodos iterativos e de simulação. Utilizando o software de simulação como parâmetro, obteve-se que o DN16 perdeu $0,32^{\circ} \mathrm{C}, \mathrm{DN} 200,17^{\circ} \mathrm{C}, \mathrm{DN} 250,11^{\circ} \mathrm{C}$ e DN32 $0,07^{\circ} \mathrm{C}$ de temperatura em um trecho de $1 \mathrm{~m}$ de comprimento. Assim, é possível conhecer o decaimento de temperatura em determinadas distâncias, fator que auxilia o projetista na escolha do tamanho da tubulação a ser projetada de acordo com o diâmetro, para uma vazão fixada.

ABSTRACT: Reticulated Polyethylene (PEX) is a flexible material used in the civil construction market in building systems of hot and cold water, and like other materials, when conducting hot water, lose temperature between the start point and the end point of an analyzed section, causing loss of energy in the form of heat to environment. This article aims to verify the behavior of temperature decay in hot water PEX pipes of different diameters and provide this information to fact sheets and designers, for this, iterative and simulation methods will be adopted. Using the simulation software as a parameter, it was obtained that the DN16 lost $0.32^{\circ} \mathrm{C}, \mathrm{DN} 200.17^{\circ} \mathrm{C}, \mathrm{DN} 250.11^{\circ} \mathrm{C}$ and DN32 $0.07^{\circ} \mathrm{C}$ of temperature in a stretch of $1 \mathrm{~m}$ of length. Thus, it is possible to know the temperature decay at certain distances, a factor that helps the designer in choosing the size of the pipe to be projected according to the diameter, for a fixed flow.

\section{CONTATO DOS AUTORES:}

${ }^{1}$ NASCIMENTO, César Henrique Britto. Estudante de Engenharia Civil. Instituto Federal de Educação, Ciência e Tecnologia de São Paulo - Campus São Paulo. cesar.nascimento@aluno.ifsp.edu.br;

${ }^{2}$ UEHARA, Takashi. Estudante de Engenharia Civil. Instituto Federal de Educação, Ciência e Tecnologia de São Paulo - Campus São Paulo. takashi.u@aluno.ifsp.edu.br.

${ }^{3}$ FERREIRA, Armando Traini. Engenheiro Civil, Doutor, Professor do Instituto Federal de Educação, Ciência e Tecnologia de São Paulo - Campus São Paulo. traini@ifsp.edu.br. 


\section{INTRODUÇÃO}

O polietileno reticulado (PEX), ou crosslinked polyethylene (XLPE) é produzido pela transformação do polietileno (normalmente de média densidade) em um material parcialmente termofixo, onde se provoca a união química das macromoléculas, reticulação, como ocorre com os materiais termofixos e elastômeros (Danieletto,2007).

Este material para tubulação vem sendo cada vez mais empregado na construção civil, consequentemente a busca por informações sobre ele também, fazendo com que diversos fabricantes divulguem catálogos e fichas técnicas sobre o produto. Entretanto, gráficos ou informações sobre o decaimento de temperatura na tubulação é um dado em falta, sendo necessário para saber estimar a perda de temperatura em um determinado trecho da tubulação.

Este Artigo tem como objetivo trazer informações sobre o decaimento de temperatura em tubulações de água quente do tipo PEX para as fichas técnicas, de modo a promover um melhor embasamento de escolha do projetista sobre o tipo de tubulação.

\section{FUNDAMENTAÇÃO TEÓRICA}

De acordo com Borgnakke e Sonntag (2012), a transferência de calor é o transporte da energia devido a diferença de temperatura entre os materiais. Esta energia pode ser transmitida por meio das colisões entre as moléculas, as quais possuem energia cinética, rotacional e vibracional. Este tipo de troca de energia por colisão é definido como condução, podendo ser expressa pela lei de Fourier, generalização baseada em uma vasta evidência experimental (INCROPERA et al,2008), conforme a Equação 1.

$Q=-K A \frac{d T}{d x}$

sendo:

$Q$, taxa de transferência de calor $(\mathrm{W})$;

$K$, condutividade térmica $\left(\mathrm{W} / \mathrm{m}^{\circ} \mathrm{C}\right)$;

$A$, área $\left(\mathrm{m}^{2}\right)$;

$T$, temperatura $\left({ }^{\circ} \mathrm{C}\right)$;

$x$, distância $(\mathrm{m})$.

Utilizando a lei de Fourier para elementos cilíndricos, conforme a Figura 1, é possível obter a Equação 2.

\section{Figura 1 - Seção transversal da tubulação}

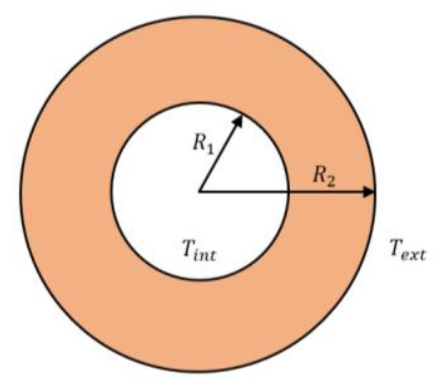

Fonte: AUTORAL (2021) 
$T_{i+1}=T_{i n t}-\frac{T_{i n t}-T_{e x t}}{R_{t} q c}$

sendo:

$T_{i+1}$, temperatura de saída do fluido no início do trecho de comprimento $L\left({ }^{\circ} \mathrm{C}\right)$;

$T_{\text {int }}$, temperatura de entrada do fluido no final do trecho de comprimento $L\left({ }^{\circ} \mathrm{C}\right)$;

$T_{\text {ext }}$, temperatura ambiente $\left({ }^{\circ} \mathrm{C}\right)$;

$R_{t}$, resistência térmica $\left({ }^{\circ} \mathrm{C} / \mathrm{W}\right)$;

$q$, vazão (g/s);

$c$, calor específico do fluido $\left(\mathrm{J} / \mathrm{g}^{\circ} \mathrm{C}\right)$.

Onde $R_{t}$, é representado pela Equação 3 .

$R_{t}=\frac{\ln \left(\frac{R_{2}}{R_{1}}\right)}{K 2 \pi L}$

sendo:

$R_{2}$, raio externo ( $\left.\mathrm{mm}\right)$;

$R_{1}$, raio interno (mm);

$L$, comprimento de análise da tubulação $(\mathrm{m})$.

A Equação 2 se apresenta de forma iterativa, sendo possível analisar a variação da temperatura em $L$ distâncias, podendo variar o material, o comprimento, a vazão e a espessura da tubulação em análise.

\section{MATERIAIS E MÉTODOS}

Os materiais abordados são todas as variações de diâmetros comerciais de tubulações do tipo PEX, conforme apresentado no Quadro 1 , com dados como condutividade térmica de 0,38 $\mathrm{W} / \mathrm{m}^{\circ} \mathrm{C}$, densidade de $945 \mathrm{Kg} / \mathrm{m}^{3}$ e calor específico de $1700 \mathrm{~J} / \mathrm{KgK}$ (AMANCO,2015).

\section{Quadro 1 - Diâmetros comerciais}

\begin{tabular}{|c|c|c|}
\hline Diâmetro comercial & Diâmetro externo $(\mathbf{m m})$ & Diâmetro interno $(\mathbf{m m})$ \\
\hline DN16 & 16 & 12,4 \\
\hline DN20 & 20 & 16,2 \\
\hline DN25 & 25 & 20,4 \\
\hline DN32 & 32 & 26,2 \\
\hline
\end{tabular}

Fonte: AMANCO (2015)

Devido ao uso de fundamentações teóricas iterativas, por análises em softwares de simulações físicas e comparações entre dados, esta pesquisa se configura no âmbito experimental.

As simulações serão realizadas pelo programa Ansys Student, o qual realiza simulações usando o método dos elementos finitos, processos numéricos e iterativos. Além disso, ao serem realizados os cálculos, é possível identificar resultados gráficos da peça em análise como um todo (ANSYS, 2021).

Para realizar a simulação, são necessárias três etapas distintas, quais sejam: o préprocessamento, solução e pós-processamento. Na primeira etapa é definida a geometria, o 
tipo de análise, as propriedades dos materiais e as condições de contorno. Na segunda é definido o tipo de solução desejada e na terceira são obtidos os resultados.

E para a utilização da fundamentação teórica iterativa, será utilizado o software Microsoft Excel, o qual realizará cálculos com a Equação 2 e entregará resultados sobre o decaimento de temperatura em $1 \mathrm{~m}$.

\section{RESULTADOS E DISCUSSÕES}

Utilizando a Equação 2, os dados do material e o trecho de $1 \mathrm{~m}$, estabeleceu-se os seguintes parâmetros: temperatura de entrada do fluido de $70^{\circ} \mathrm{C}$, temperatura ambiente de $20{ }^{\circ} \mathrm{C}$, velocidade do fluido de $3 \mathrm{~m} / \mathrm{s}$, calor específico da água de $4,18 \mathrm{~J} / \mathrm{g}^{\circ} \mathrm{C}$ (WOLFRAMALPHA COMPUTATIONAL INTELLIGENCE, 2021), obtendo-se o Quadro 2.

Quadro 2 - Resultados iterativos do Excel

\begin{tabular}{|c|c|c|c|c|c|c|c|}
\hline \multicolumn{2}{|c|}{ DN16 } & \multicolumn{2}{c|}{ DN20 } & \multicolumn{2}{c|}{ DN25 } & \multicolumn{2}{c|}{ DN32 } \\
\hline $\mathbf{L}(\mathbf{m})$ & $\boldsymbol{T}_{\boldsymbol{i + 1}}\left(\mathbf{~}^{\circ} \mathbf{C}\right)$ & $\mathbf{L}(\mathbf{m})$ & $\boldsymbol{T}_{\boldsymbol{i + 1}}\left({ }^{\circ} \mathbf{C}\right)$ & $\mathbf{L}(\mathbf{m})$ & $\boldsymbol{T}_{\boldsymbol{i + 1}}\left({ }^{\circ} \mathbf{C}\right)$ & $\mathbf{L}(\mathbf{m})$ & $\boldsymbol{T}_{\boldsymbol{i + 1}}\left({ }^{\circ} \mathbf{C}\right)$ \\
\hline 0,0 & 70,00 & 0,0 & 70,00 & 0,0 & 70,00 & 0,0 & 70,00 \\
\hline 0,1 & 69,96 & 0,1 & 69,98 & 0,1 & 69,99 & 0,1 & 69,99 \\
\hline 0,2 & 69,91 & 0,2 & 69,95 & 0,2 & 69,97 & 0,2 & 69,98 \\
\hline 0,3 & 69,87 & 0,3 & 69,93 & 0,3 & 69,96 & 0,3 & 69,97 \\
\hline 0,4 & 69,82 & 0,4 & 69,90 & 0,4 & 69,94 & 0,4 & 69,96 \\
\hline 0,5 & 69,78 & 0,5 & 69,88 & 0,5 & 69,93 & 0,5 & 69,95 \\
\hline 0,6 & 69,73 & 0,6 & 69,85 & 0,6 & 69,91 & 0,6 & 69,95 \\
\hline 0,7 & 69,69 & 0,7 & 69,83 & 0,7 & 69,90 & 0,7 & 69,94 \\
\hline 0,8 & 69,64 & 0,8 & 69,81 & 0,8 & 69,88 & 0,8 & 69,93 \\
\hline 0,9 & 69,60 & 0,9 & 69,78 & 0,9 & 69,87 & 0,9 & 69,92 \\
\hline 1,0 & 69,55 & 1,0 & 69,76 & 1,0 & 69,85 & 1,0 & 69,91 \\
\hline
\end{tabular}

Fonte: AUTORAL (2021)

Após a obtenção destes dados, foi gerado o gráfico da Figura 2, no qual é visto a diferença do decaimento de temperatura em $1 \mathrm{~m}$ de comprimento entre os diâmetros comerciais, de modo que a tubulação DN16 possuiu uma perda de 0,64\%, DN20 de 0,34\%, DN25 de 0,21\% e DN32 de $0,13 \%$ em relação à temperatura de entrada de $70^{\circ} \mathrm{C}$. 


\section{Figura 2 - Gráfico de decaimento de temperatura}

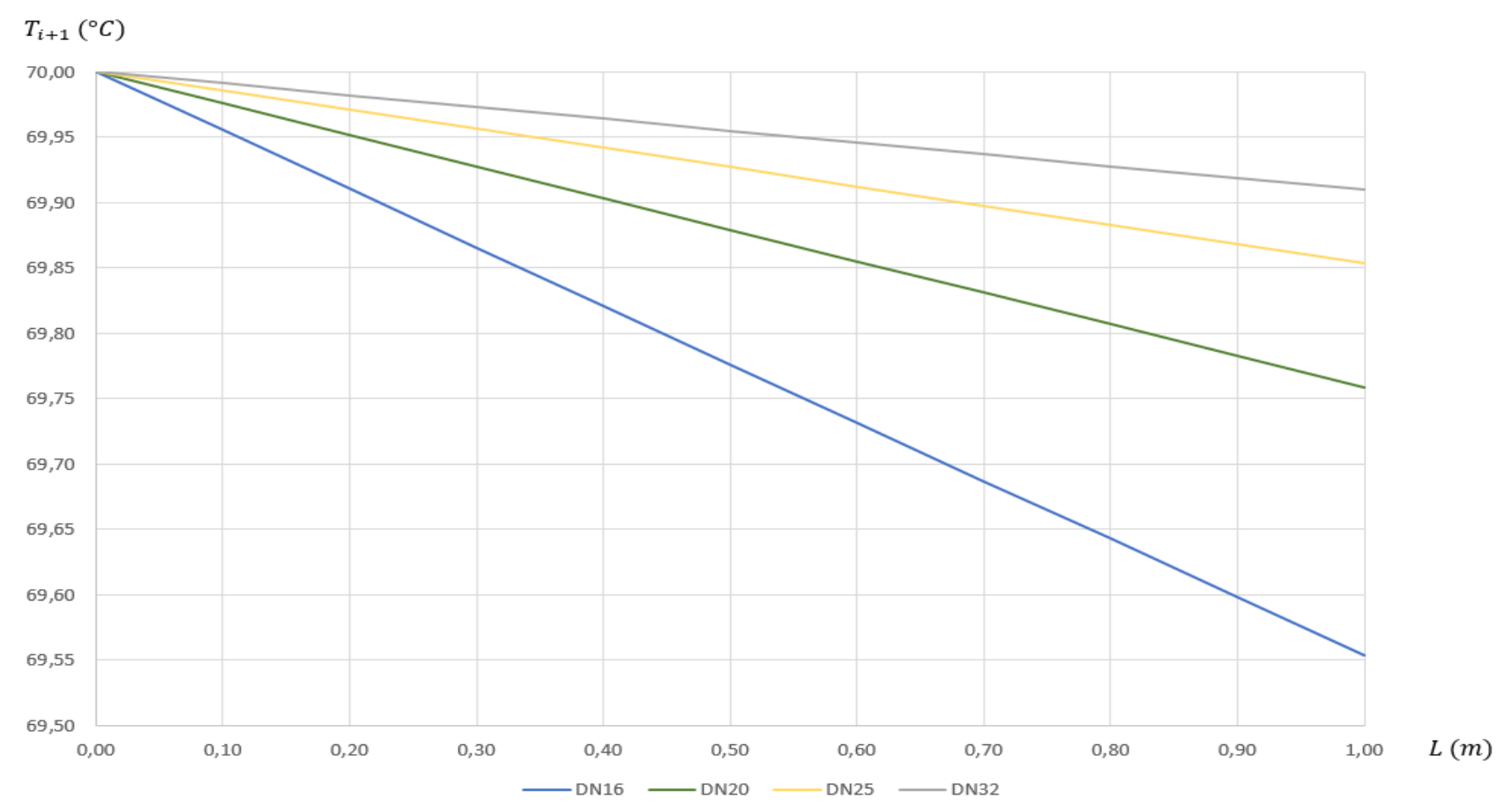

Fonte: AUTORAL (2021)

Realizado esse processo, foi efetuada a etapa de simulações, na qual foram inseridas as informações sobre as tubulações PEX no software.

Todas as simulações realizadas seguiram o modelo standard com viscosidade k-ômega, o qual, é baseado no modelo Wilcox, cujo prevê taxas de espalhamento de fluxo de cisalhamento livre que estão de acordo com as medições para ondas distantes, camadas de mistura e jatos planos, redondos e radiais e, portanto, é aplicável a fluxos delimitados por parede (ANSYS,2009) e segundo Georgiadis (2006), "nas análises de simulação numérica o modelo de turbulência k-ômega é muito usado, pois apresenta uma exatidão das predições".

Além disso, os cálculos foram computados a partir do dado inicial inlet (temperatura de entrada do fluido) e finalizados no outlet (temperatura de saída do fluido após o comprimento de $1 \mathrm{~m})$. 
Figura 3 - Gráfico de iterações para 1m de tubulação - (PEX DN16)

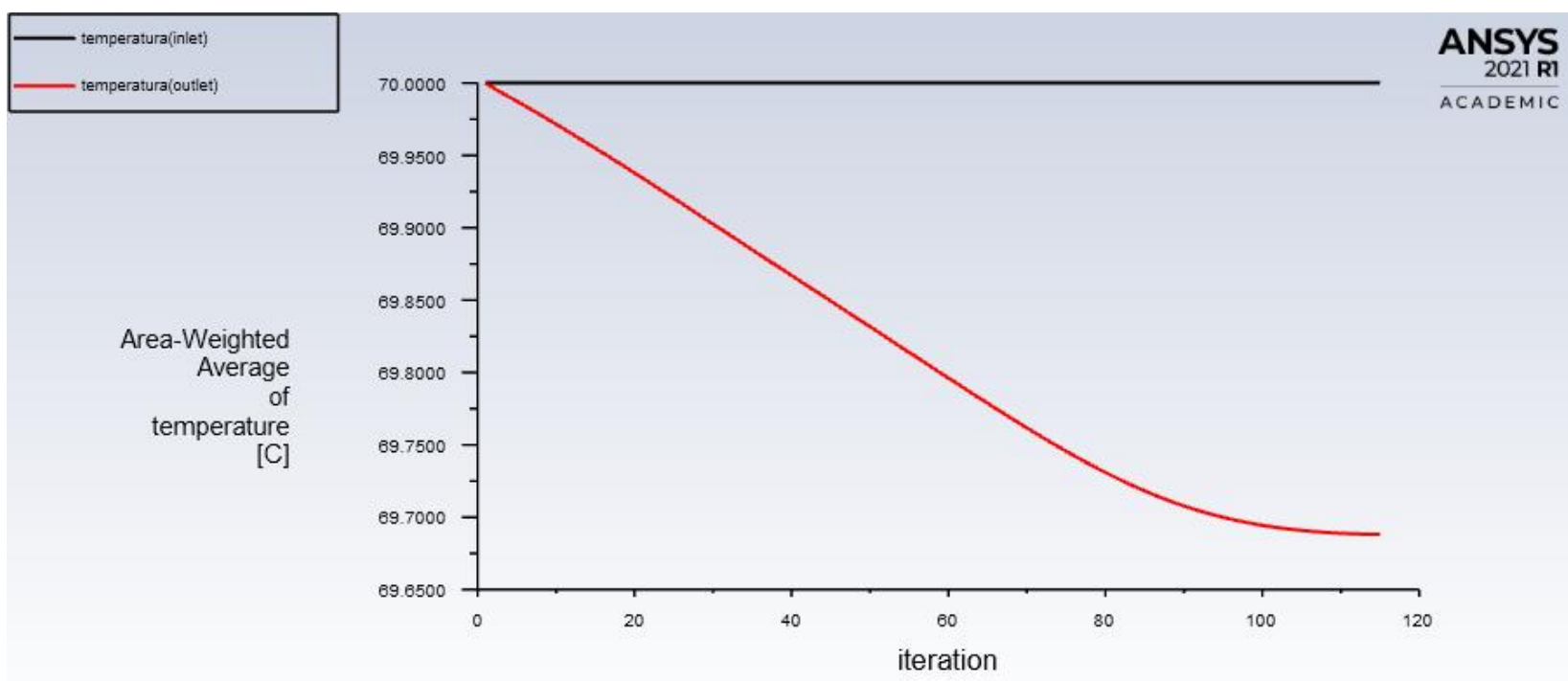

Fonte: AUTORAL (2021)

Todas as simulações dos diâmetros comerciais apresentaram gráficos similares ao da Figura 3, a qual apresenta a quantidade de iterações para a convergência da temperatura após $1 \mathrm{~m}$ no eixo das abscissas e no eixo das ordenadas os valores de temperatura.

Desse modo, todos os dados obtidos foram organizados no Quadro 3.

Quadro 3 - Temperaturas dos fluidos nos diâmetros comerciais após $1 \mathrm{~m}$ de tubulação (Ansys)

\begin{tabular}{|c|c|}
\hline Diâmetro comercial & Temperatura $\left({ }^{\circ} \mathrm{C}\right)$ \\
\hline DN16 & 69,68 \\
\hline DN20 & 69,83 \\
\hline DN25 & 69,89 \\
\hline DN32 & 69,93 \\
\hline
\end{tabular}

Fonte: AUTORAL (2021)

Conforme os dados apresentados, a tubulação DN16 possuiu uma perda de 0,46\%, DN20 de $0,24 \%$, DN25 de 0,16\% e DN32 de 0,10\% em relação à temperatura inicial de $70^{\circ} \mathrm{C}$. Desta maneira, é possível relacionar os decaimentos pela Equação 2 e os obtidos em simulação, sendo que os valores de perdas de temperatura destes se apresentam de maneira aproximada, conforme o Quadro 4. 
Quadro 4 - Relação entre perdas de temperatura

\begin{tabular}{|c|c|c|}
\hline Diâmetro comercial & $\begin{array}{c}\text { Perda de temperatura em 1 m (\%) } \\
\text { Equação 2 }\end{array}$ & $\begin{array}{c}\text { Perda de temperatura em 1 m (\%) } \\
\text { Ansys }\end{array}$ \\
\hline DN16 & 0,64 & 0,46 \\
\hline DN20 & 0,34 & 0,24 \\
\hline DN25 & 0,21 & 0,16 \\
\hline DN32 & 0,13 & 0,10 \\
\hline
\end{tabular}

Fonte: AMANCO (2015)

Desta forma, é possível avaliar que, tanto no Ansys Student quanto na Equação 2, os menores diâmetros possuem maiores decaimentos de temperatura, assim como é previsto na lei de Fourier (Equação 1), na qual é informado que a taxa de transferência de calor é inversamente proporcional à distância.

Após essa análise, foi feita a construção do gráfico de perda de temperatura do fluido inserido na tubulação mediante a uma coordenação por cores na Figura 3.

Figura 3 - Decaimento de temperatura do fluido em 1m de tubulação - (PEX DN16)

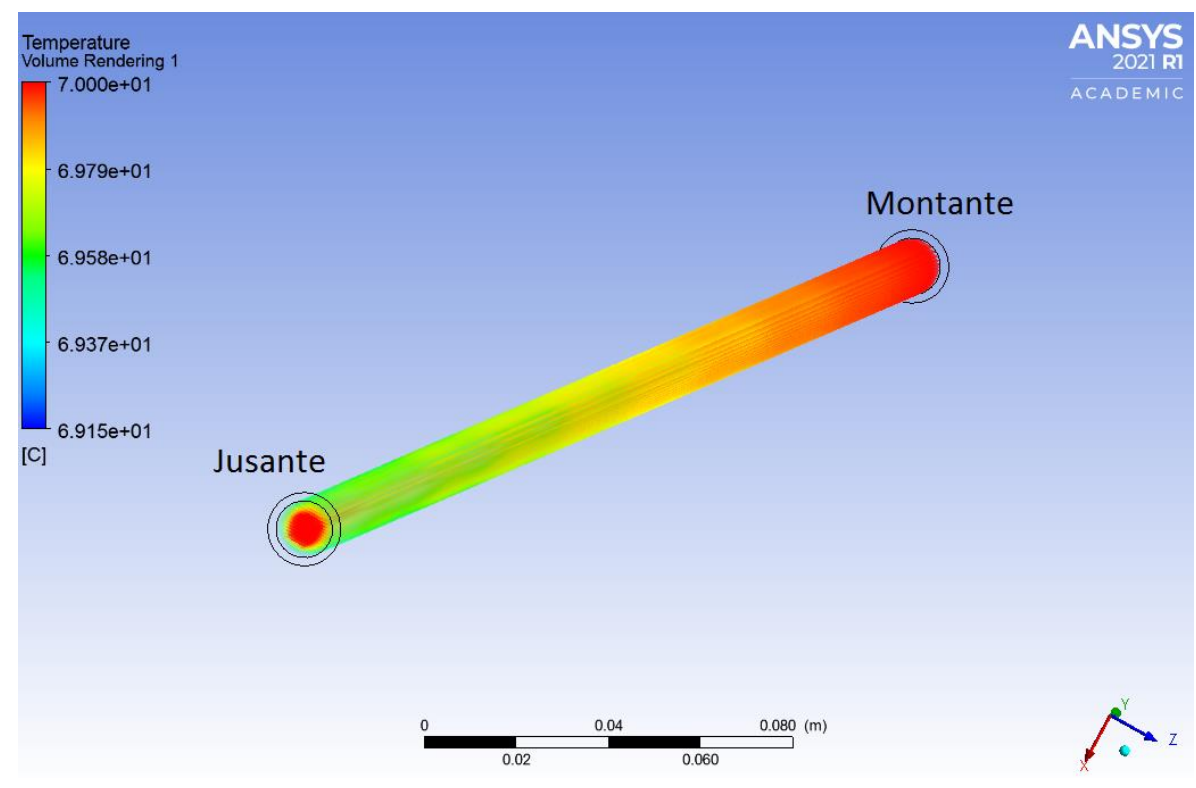

Fonte: AUTORAL (2021)

Por essa nomenclatura, é possível conferir a variação da temperatura em cada ponto da extensão, de modo que as áreas externas possuem maiores perdas de temperatura e as áreas internas, menores, característica descrita pela lei de Fourier (Equação 1).

\section{CONCLUSÃO}

Devido a importância da informação sobre o decaimento de temperatura em tubulações para a construção civil, o artigo visa auxiliar os projetistas e contribuir com dados simulados no software Ansys Student para superar esta limitação apresentada em catálogos e fichas técnicas deste produto. 
Utilizando o Ansys Student como parâmetro é possível avaliar o decaimento de temperatura máximo $\left(0,32^{\circ} \mathrm{C}\right)$ no $\mathrm{DN} 16$ e mínimo $\left(0,07^{\circ} \mathrm{C}\right)$ para o DN32 em um trecho de $1 \mathrm{~m}$ de comprimento. Conhecendo os valores indicados pelo Ansys Student e os da Lei de Fourier (Eq. 2) é possível verificar que os dados decaem de forma linear, desse modo, sabendo o quanto o dado variou em $1 \mathrm{~m}$ é possível prever sua perda de temperatura em " $\mathrm{n}$ " metros, fator que auxilia o projetista na escolha do comprimento da tubulação a ser projetada de acordo com o diâmetro.

\section{REFERÊNCIAS}

AMANCO. Manual técnico - PEX, 2015. Disponível em: $<$ http://assets.production.amanco.com.br.s3.amazonaws.com/uploads/collapse/file/49/ManualPEX-2015-WEB-FINAL.pdf >. Data de acesso: 14 de jan. 2021.

ANSYS. ANSYS FOR STUDENTS, 2021. Disponível em: <https://www.ansys.com/academic/students>. Data de acesso: 25 de mai. 2021.

ANSYS. Standard k-ômega model, 2009. Disponível em: < https://www.afs.enea.it/project/neptunius/docs/fluent/html/th/node66.htm>. Data de acesso: $20 \mathrm{de}$ set. 2021.

BORGNAKKE, C. SONNTAG. R. E. Fundamentals of thermodynamics. 8a Edição. Michigan: John Wiley \& Sons, 2012. 916p.

DANIELETTO, J. R. B. Manual de Tubulações de Polietileno e Polipropileno. São Paulo: Linha Aberta, 2007. 524p.

GEORGIADIS, N. J. Evaluation of Modified Two-Equation Turbulence Models for Jet Flow Predictions. AIAA J, 2006, 3114p.

INCROPERA, F. P. et al. Fundamentos de transferência de calor e de massa. 6. Ed. Rio de Janeiro: LTC, 2008. 645p.

WOLFRAMALPHA COMPUTATIONAL INTELLIGENCE . SPECIFIC HEAT OF WATER, 2021. Disponível em: <https://www.wolframalpha.com/input/?i=specific+heat+of+water>. Data de acesso: 30 de mai. 2021. 